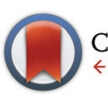

CrossMark

Cite this: Polym. Chem., 2016, 7, 7522

Received 8th October 2016, Accepted 14th November 2016 DOI: $10.1039 / c 6 p y 01759 b$ www.rsc.org/polymers

\section{Synthesis and chiroptical properties of a $\pi$-conjugated polymer containing glucose-linked biphenyl units in the main chain capable of folding into a helical conformation $\uparrow$}

\author{
Tomoyuki Ikai, * Syo Shimizu, Seiya Awata, Tomoya Kudo, Takayuki Yamada, \\ Katsuhiro Maeda and Shigeyoshi Kanoh
}

\begin{abstract}
An optically active $\pi$-conjugated polymer (poly-9) containing glucose-linked biphenyl units in the main chain was synthesized through the copolymerization of 5,5'-diiodo-2,2'-bithiophene and a new diethynyl compound, whose molecular design has taken inspiration from naturally occurring ellagitannins. The chiroptical properties of poly-9 were investigated in solution and the solid state. The absorption and circular dichroism spectra of poly-9 were clearly changed depending on the solvents due to the conformational alteration within a single polymer chain. Because a corresponding model molecule and polymer did not show marked solvent-dependent spectral changes, the backbone conformation of poly-9 is considered to be capable of interconverting between random-coil and preferred-handed helical states in response to the exterior environment. We also found that poly- 9 exhibited efficient circularly polarized luminescence with a green color, whose dissymmetry factor reached $1.9 \times 10^{-2}$ when the backbone was folded into the helical conformation in the film state.
\end{abstract}

\section{Introduction}

The development of valuable functional materials derived from abundant and renewable resources has been recognized as one of the most important aspects to break out of the excessive dependence on dwindling fossil fuels and to realize a sustainable society. In nature, there are a variety of organic compounds with unique chemical structures, some of which can still be obtained only from nature. The incorporation of interesting structural features of natural products into molecular designs is very attractive for material development because it opens up the possibility of creating novel materials that would be otherwise unimaginable. Naturally occurring ellagitannin is a series of natural compounds containing one or more biphenyl units linked to a sugar moiety. Two structural examples of ellagitannin, tellimagrandin I and pedunculagin, are shown in Chart 1; these compounds are known to be present in fruits of Eucalyptus globulus and pericarps of Punica granatum. ${ }^{1}$ These molecules are optically active, not only because of the

Graduate School of Natural Science and Technology, Kanazawa University, Kakuma-machi, Kanazawa 920-1192, Japan.E-mail: ikai@se.kanazawa-u.ac.jp; Fax: +81-76-2344781; Tel: $+81-76-2344781$

$\dagger$ Electronic supplementary information (ESI) available: Detailed experimental procedures, characterization of monomers/polymers and additional spectroscopic, chromatographic and computational data. See DOI: 10.1039/c6py01759b

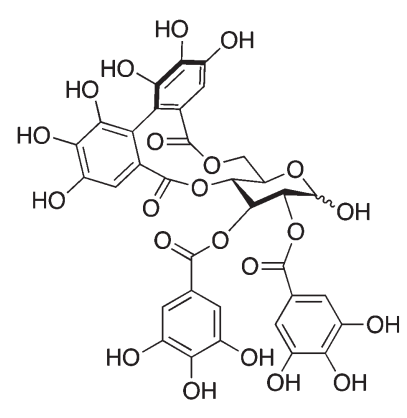

Tellimagrandin I

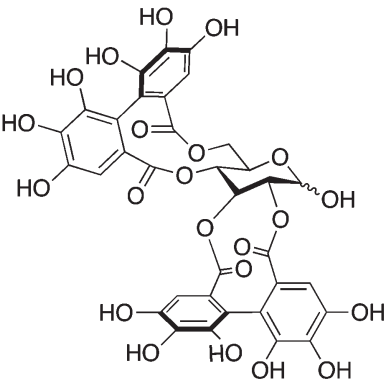

Pedunculagin
Chart 1

chiral centers in the sugar unit, but also due to the chiral axis derived from the biphenyl unit. Despite its unique chiral structure, ellagitannin has not yet been utilized as a building unit for chiral functional materials.

Synthetic oligomers and polymers capable of folding into a well-defined compact conformation in solution are known as foldamers. ${ }^{2}$ These molecules adopt folded states only in particular environments, in which intramolecular interactions, such as hydrogen bonding, coordination bonding and lyophobic interactions, work efficiently over the entire molecular strand. A representative example is the oligo $(m$-phenylene 


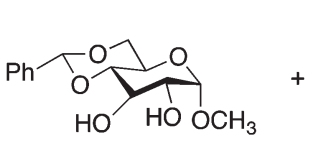

1
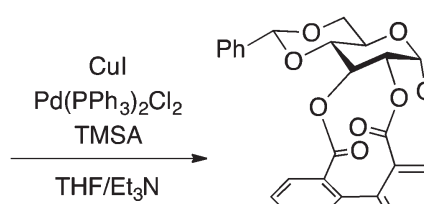

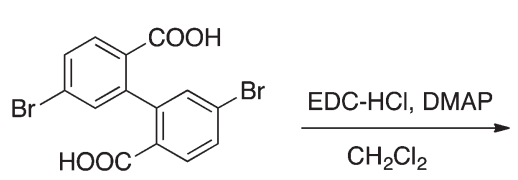

2
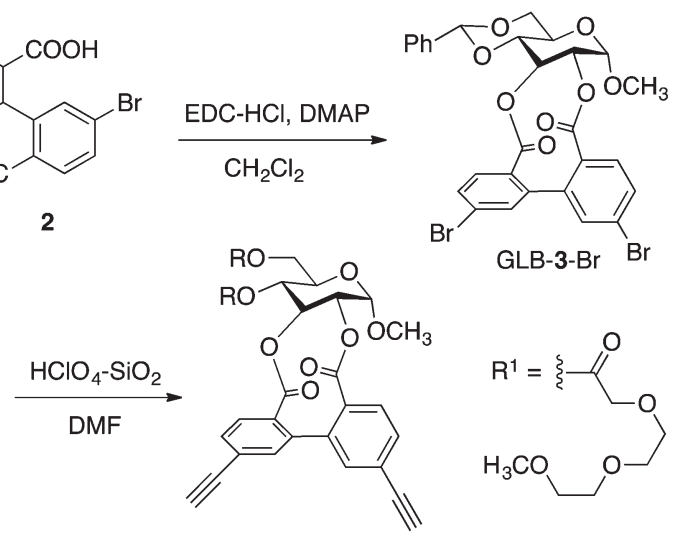

GLB-3-Br Br

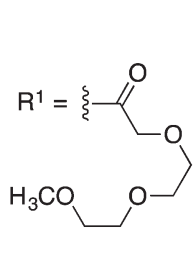

$\mathrm{R}^{1}-\mathrm{OH}, \mathrm{EDC}-\mathrm{HCl}, \square$ GLB-6: $\mathrm{R}=\mathrm{H}$

DMAP, $\mathrm{CH}_{2} \mathrm{Cl}_{2} \longrightarrow$ GLB-7: $\mathrm{R}=\mathrm{R}^{1}$

Scheme 1 Synthesis of GLB-7 bearing a glucose-linked biphenyl unit.

ethynylene)s reported by Moore and co-workers. ${ }^{3}$ In these systems, lyophobic interactions play a critical role in promoting helical folding behavior; the oligomers adopt helical conformations in polar solvents such as acetonitrile, but unfold into randomcoils in chloroform. Foldamers consisting of only achiral building blocks form an equimolar mixture of right- and lefthanded helices when folding into helical states. The incorporation of optically active components into the molecular structures, for example in the main chains, ${ }^{4}$ side chains ${ }^{5}$ or chain terminus, ${ }^{4 a, 6}$ is a typical approach for selectively inducing excess one-handed helical states. Up to now, a variety of structural motifs have been employed for producing optically active foldamers, ${ }^{2 b, c, e-g, 7}$ and their application as chiral functional materials, associated with chiral sensors, ${ }^{8}$ asymmetric catalysis ${ }^{9}$ and chiral stationary phase, ${ }^{10}$ has been reported.

We anticipated that the preferred-handed twisted biphenyl structure of ellagitannin could be used as a new structural motif for the development of $\pi$-conjugated foldamers. Herein, we designed a diethynyl monomer (GLB-7 in Scheme 1) bearing a glucose-linked biphenyl unit that is chirally twisted as in the ellagitannins. Copolymerization of GLB-7 with a bithiophenebased cross-coupling partner provided a $\pi$-conjugated polymer that showed a helix-to-random coil transition in response to the solvent environment. To the best of our knowledge, this is the first example of a bio-inspired foldamer created by taking advantage of a structural feature of the ellagitannins. We also report on the preliminary application of this polymer in circularly polarized luminescence (CPL) materials.

\section{Experimental section}

\section{Materials}

Anhydrous solvents (dichloromethane, chloroform, acetonitrile and tetrahydrofuran (THF)) and common organic solvents were purchased from Kanto Kagaku (Tokyo, Japan). [2-(2Methoxyethoxy)ethoxy]acetic acid was from Tokyo Kasei Kogyo
(TCI) (Tokyo, Japan). 5,5'-Diiodo-2,2'-bithiophene (8) and copper(I) iodide (CuI) were from Sigma-Aldrich (St Louis, MO, USA). 1-Ethyl-3-(3-dimethylaminopropyl)carbodiimide hydrochloride (EDC-HCl) and N,N-dimethyl-4-aminopyridine (DMAP) were purchased from Wako Pure Chemical Industries (Osaka, Japan). Tetrakis(triphenylphosphine)palladium(0) $\left(\mathrm{Pd}\left(\mathrm{PPh}_{3}\right)_{4}\right)$ was purchased from Nacalai (Kyoto, Japan). Diisopropylamine (DIPA) was obtained from Kishida (Osaka, Japan). Chiralpak IA $(25 \mathrm{~cm} \times 0.46 \mathrm{~cm}$ (i.d.) $)$ was purchased from Daicel (Tokyo, Japan). Details on the synthesis of GLB-6, mono-10 and poly-11 are described in the ESI. $\dagger$

\section{Synthesis}

Synthesis of GLB-7. To a solution of GLB-6 (0.54 g, $1.2 \mathrm{mmol})$, [2-(2-methoxyethoxy)ethoxy]acetic acid $(0.47 \mathrm{~g}$, $2.6 \mathrm{mmol})$ and DMAP $(0.32 \mathrm{~g}, 2.6 \mathrm{mmol})$ in dichloromethane $(12 \mathrm{~mL})$ was added EDC- $\mathrm{HCl}(0.51 \mathrm{~g}, 2.6 \mathrm{mmol})$ at $0{ }^{\circ} \mathrm{C}$ under a nitrogen atmosphere. After stirring at room temperature for $8 \mathrm{~h}$, the reaction system was diluted with dichloromethane and the solution was washed with $1 \mathrm{~N} \mathrm{HCl}$ aqueous solution and water, and then dried over $\mathrm{Na}_{2} \mathrm{SO}_{4}$. After filtration, the solvent was removed by evaporation and the crude product was purified by silica gel chromatography using ethyl acetate as the eluent to give the desired product as a white solid $(0.70 \mathrm{~g}, 76 \%$ yield). Mp: $50.2-50.5{ }^{\circ} \mathrm{C}$. $[\alpha]_{\mathrm{D}}^{25}+15.5$ (c 1.0, $\left.\mathrm{CHCl}_{3}\right) .{ }^{1} \mathrm{H}$ NMR $\left(500 \mathrm{MHz}, \mathrm{CDCl}_{3}, \mathrm{rt}\right): \delta 7.65-7.50(\mathrm{~m}, 4 \mathrm{H}, \mathrm{ArH}), 7.46(\mathrm{~d}, J=8.0$ $\mathrm{Hz}, 1 \mathrm{H}, \operatorname{ArH}), 7.32$ (d, $J=8.0 \mathrm{~Hz}, 1 \mathrm{H}, \operatorname{ArH}), 5.63$ (t, $J=9.5 \mathrm{~Hz}$, $1 \mathrm{H}, \mathrm{CH}), 5.40-5.30(\mathrm{~m}, 2 \mathrm{H}, \mathrm{CH}), 5.02-4.93(\mathrm{~m}, 1 \mathrm{H}, \mathrm{CH})$, 4.43-4.05 (m, 7H, $\left.\mathrm{CH}_{2}, \mathrm{CH}\right), 3.80-3.47\left(\mathrm{~m}, 19 \mathrm{H}, \mathrm{CH}_{2}, \mathrm{CH}\right.$, $\left.\mathrm{OCH}_{3}\right), 3.38\left(\mathrm{~s}, 3 \mathrm{H}, \mathrm{OCH}_{3}\right), 3.37\left(\mathrm{~s}, 3 \mathrm{H}, \mathrm{OCH}_{3}\right), 3.21(\mathrm{~s}, 2 \mathrm{H}$, $\mathrm{C} \equiv \mathrm{CH}) .{ }^{13} \mathrm{C}$ NMR (125 MHz, $\left.\mathrm{CDCl}_{3}, \mathrm{rt}\right): \delta 170.30,169.18$, 168.25, 168.08, 136.91, 136.72, 134.50, 134.47, 132.76, 132.69, $131.73,126.63,126.43,125.44,97.40,82.30,79.95,74.97$, $74.02,72.02,71.98,71.08,70.70,70.65,68.43,67.81,67.25$, 61.90, 59.15, 59.13, 55.85. IR $\left(\mathrm{KBr}, \mathrm{cm}^{-1}\right): 2109(\mathrm{C} \equiv \mathrm{C}), 1752$ $(\mathrm{C}=\mathrm{O})$. Calcd for $\mathrm{C}_{39} \mathrm{H}_{44} \mathrm{O}_{16}$ : C, 60.93; H, 5.77; Found: C, $60.96 ; \mathrm{H}, 5.66$. 


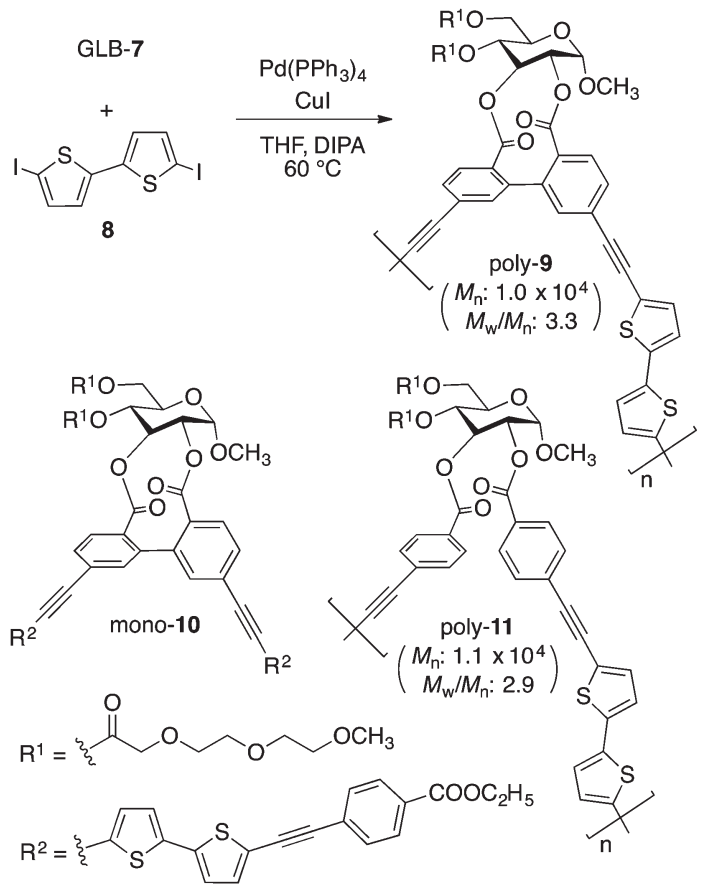

Fig. 1 Synthesis of poly-9 and structures of mono-10 and poly-11.

Synthesis of poly-9. Copolymerization of GLB-7 with 8 by the Sonogashira-Hagihara cross coupling was carried out using $\mathrm{Pd}\left(\mathrm{PPh}_{3}\right)_{4}$ as a catalyst in a dry Schlenk flask under a nitrogen atmosphere (Fig. 1). To a solution of GLB-7 (0.48 g, $0.62 \mathrm{mmol}), \mathrm{Pd}\left(\mathrm{PPh}_{3}\right)_{4}(43 \mathrm{mg}, 0.037 \mathrm{mmol})$ and $\mathrm{CuI}(36 \mathrm{mg}$, $0.19 \mathrm{mmol}$ ) in degassed THF/DIPA (5/1, v/v) (21 mL) was added 8 (0.26 g, $0.62 \mathrm{mmol})$. The solution was stirred at $60{ }^{\circ} \mathrm{C}$ for $12 \mathrm{~h}$. After cooling to room temperature, the reaction mixture was poured into a large amount of hexane, and the resulting polymer was collected by centrifugation, washed with ethanol, and dried in vacuo to yield poly-9 as an orange solid (0.49 g, 85\%). ${ }^{1} \mathrm{H}$ NMR (400 MHz, $\left.\mathrm{CDCl}_{3}, 55^{\circ} \mathrm{C}\right): \delta 7.70-7.40$ (m, 5H, ArH), 7.34 (d, $J=7.6 \mathrm{~Hz}, 1 \mathrm{H}, \mathrm{ArH}), 7.20$ (br, 2H, ArH), 7.10 (br, 2H, ArH), 5.64 (t, $J=9.6 \mathrm{~Hz}, 1 \mathrm{H}, \mathrm{CH}), 5.40-5.20(\mathrm{~m}$, $2 \mathrm{H}, \mathrm{CH}), 5.05-4.90(\mathrm{~m}, 1 \mathrm{H}, \mathrm{CH}), 4.45-4.35(\mathrm{~m}, 1 \mathrm{H}, \mathrm{CH})$, 4.30-4.15 (m, 5H, $\left.\mathrm{CH}_{2}, \mathrm{CH}\right), 4.08$ (d, $\left.J=9.2 \mathrm{~Hz}, 1 \mathrm{H}, \mathrm{CH}\right)$, 3.80-3.60 (m, 12H, CH $)$, 3.58-3.40 (m, 7H, $\left.\mathrm{CH}_{2}, \mathrm{OCH}_{3}\right), 3.36$ (d, $\left.J=5.6 \mathrm{~Hz}, 6 \mathrm{H}, \mathrm{OCH}_{3}\right)$. IR $\left(\mathrm{KBr}, \mathrm{cm}^{-1}\right): 2197(\mathrm{C} \equiv \mathrm{C}), 1752$ $(\mathrm{C}=\mathrm{O})$. Calcd for $\mathrm{C}_{47} \mathrm{H}_{46} \mathrm{O}_{16} \mathrm{~S}_{2} \cdot 1.4 \mathrm{H}_{2} \mathrm{O}: \mathrm{C}, 59.04 ; \mathrm{H}, 5.14$. Found: C, 58.98; H, 5.04.

\section{Instruments}

NMR spectra were recorded on a JNA-LA 400 (JEOL, Tokyo, Japan) (400 MHz for ${ }^{1} \mathrm{H}$ ) or a JNM-ECA 500 (JEOL) (500 MHz for ${ }^{1} \mathrm{H}, 125 \mathrm{MHz}$ for ${ }^{13} \mathrm{C}$ ) spectrometer in $\mathrm{CDCl}_{3}$ using tetramethylsilane as the internal standard. Melting points were measured on Yanako melting point apparatus and were uncorrected. IR spectra were obtained using a JASCO (Hachioji, Japan) Fourier Transform IR-460 spectrophotometer with a $\mathrm{KBr}$ pellet. The molecular weights and distributions of the polymers were estimated using size-exclusion chromatography
(SEC) equipped with a TSKgel Multipore $\mathrm{H}_{\mathrm{XL}}-\mathrm{M}$ column (Tosoh, Tokyo, Japan), a JASCO PU-2080 Plus high-performance liquid chromatography (HPLC) pump and a JASCO UV-970 UV/VIS detector at $254 \mathrm{~nm}$, where chloroform was used as the eluent. The molecular weight calibration curve was obtained with polystyrene standards (Tosoh). The optical rotation was measured at $25{ }^{\circ} \mathrm{C}$ with a JASCO P-1030 polarimeter. Absorption and circular dichroism (CD) spectra in solution states were measured using a JASCO V-570 and a JASCO J-725 spectrometers, respectively, with a $0.10,1.0$, or $10 \mathrm{~mm}$ quartz cell. The temperature was controlled using a JASCO ETC-505T (absorption spectroscopy) and JASCO PTC-348WI apparatus (CD spectroscopy). Photoluminescence (PL) and circularly polarized luminescence (CPL) spectra were recorded at room temperature on a JASCO CPL-300 spectrometer. Spincoated films prepared on quartz substrates from a chloroform or a chloroform/acetonitrile $(60 / 40, \mathrm{v} / \mathrm{v})$ solution $\left(10 \mathrm{mg} \mathrm{mL} \mathrm{mL}^{-1}\right)$ were used for solid-state spectral measurements. Elemental analyses were performed at the Research Institute for Instrumental Analysis of Advanced Science Research Center, Kanazawa University, Kanazawa, Japan.

\section{Results and discussion}

\section{Synthesis}

Scheme 1 shows the synthetic route to GLB-7, which was synthesized in five steps using a glucose derivative (1) with two hydroxy groups as the starting material. Each reaction proceeded well to afford the corresponding product in moderate to excellent yields, with an overall yield of $43 \%$ (see the ESI $\dagger$ ). On the basis of a ${ }^{1} \mathrm{H}$ NMR assignment of a previously reported artificial ellagitannin bearing a biphenyl unit with $(S)$ - or $(R)$ configuration, ${ }^{11}$ GLB-7 was presumed to contain two diastereomers with opposite axial chirality (GLB-7S and GLB-7R in Chart 2), and their diastereomeric ratio was determined to be $94: 6$ in favor of GLB-7S (ESI Fig. S1A $\dagger$ ). These isomers were efficiently separated from each other by chiral HPLC using
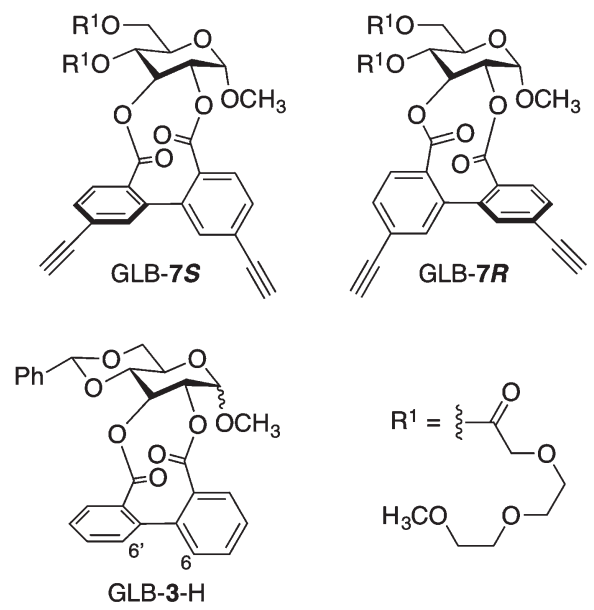

Chart 2 
Chiralpak IA as the chiral stationary phase, as shown in Fig. S2A. $\dagger$ The CD spectra of each component, which were recorded using the stop-flow HPLC-CD analysis, revealed that the two isomers show mirror-image-like CD patterns in the absorption region of the biphenyl units (ESI Fig. S2B †). This is further evidence that the twist senses of the biphenyl units were opposite to each other although their twist angles would not be the same. Interestingly, when each isolated component recovered from the HPLC system was reinjected onto the column under the same conditions, two clear peaks derived from the diastereomers appeared again in the chromatograms (ESI Fig. S3†), whose retention times were almost the same as those in the original separation. Although the axial chirality of an analogous glucose derivative (GLB-3-H in Chart 2) without any substituents at the 6,6'-positions of the biphenyl unit has so far been considered to be a static feature, ${ }^{12}$ our results clearly indicate that the twist handedness of the biphenyl unit in GLB-7 was dynamic and that GLB-7 was in a state of dynamic equilibrium between the two diastereomers with opposite axial chirality, GLB-7S and GLB-7 $\boldsymbol{R}^{13}$

GLB-7 was copolymerized with 8 through a SonogashiraHagihara coupling using a palladium catalyst in a THF/DIPA mixture $(5 / 1, \mathrm{v} / \mathrm{v})$ at $60{ }^{\circ} \mathrm{C}$ (Fig. 1 ). The resulting polymer was purified by successive washing with ethanol and hexane to remove low molecular weight oligomers and catalytic residues.
Optically active poly-9 containing glucose-linked biphenyl units in the main chain was obtained in a relatively good yield (85\%), and its molecular weight $\left(M_{n}\right)$ and the degree of polymerization were estimated by SEC to be approximately $1.0 \times 10^{4} \mathrm{~g} \mathrm{~mol}^{-1}$ and $c a .11$, respectively. We confirmed that the ${ }^{1} \mathrm{H}$ NMR spectrum of the obtained poly- 9 contained a series of signals derived from the GLB-7S and GLB-7 $\boldsymbol{R}$ units, and the compositional ratio was almost the same as the diastereomeric ratio of the monomer (ESI Fig. S1B †). This clearly means that the axial chirality of the biphenyl units in the polymer backbone is biased in favor of the $(S)$-configuration, although it remains unclear whether the axial chirality possesses a dynamic nature. We also prepared two model compounds related to poly-9 (Fig. 1 and Scheme $\mathbf{S} 1 \dagger$ ): one is a monomeric model (mono-10) containing bithiophene-based $\pi$-conjugated units at the 2- and 3-positions of the glucose unit, and the other is a polymeric model (poly-11) without a covalent linkage between the phenyl rings.

\section{Chiroptical properties in solutions}

Fig. 2A and B show the CD and absorption spectra of poly-9 and mono-10, respectively. In chloroform, poly-9 showed a Cotton effect in the absorption region of the polymer backbone, and its intensity and pattern were quite similar to those of mono-10. Their split-type CD patterns with the positive first

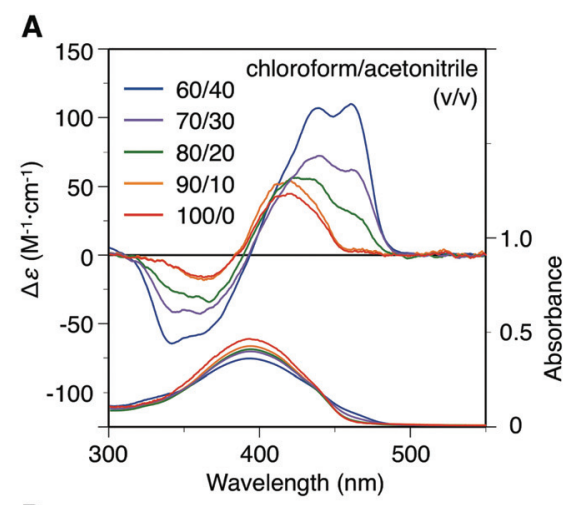

D

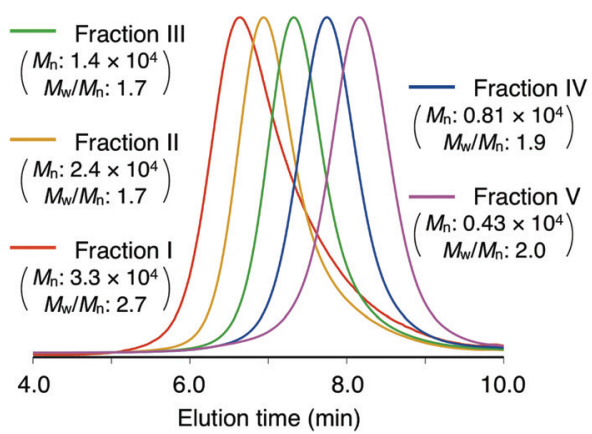

B
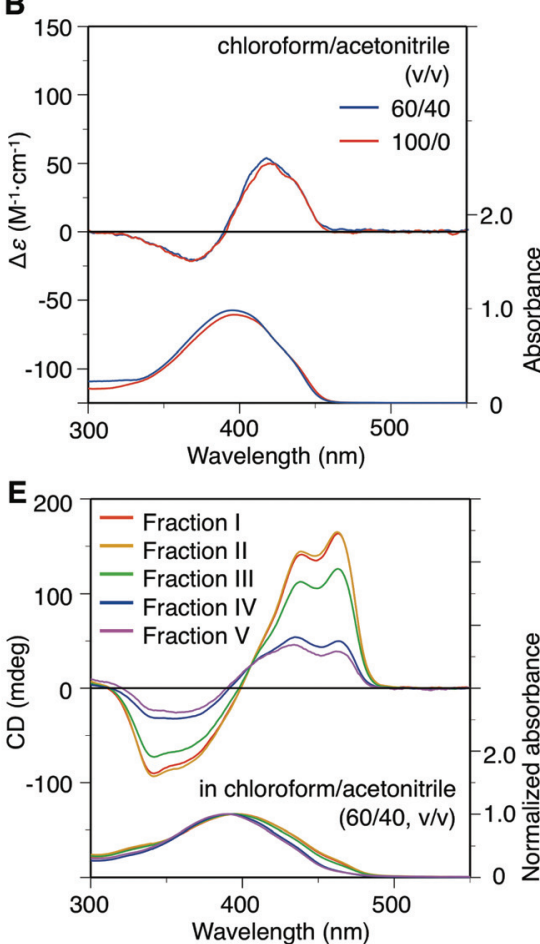

C

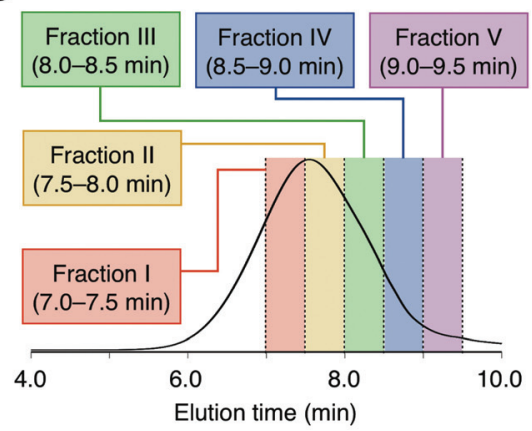

F

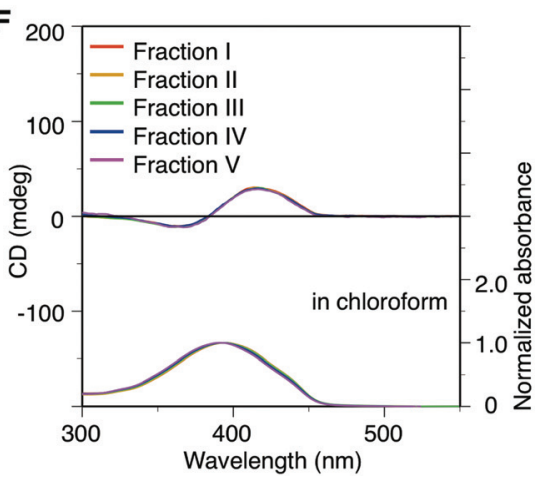

Fig. 2 (A, B) CD and absorption spectra of poly-9 (A) and mono-10 (B) in chloroform/acetonitrile $(100 / 0-60 / 40, v / v)$ at $25^{\circ} \mathrm{C}$. [Glucose unit] $=1.0$ $\times 10^{-4} \mathrm{M}$. (C, D) SEC traces of the as-synthesized poly-9 $\left(M_{\mathrm{n}}: 1.0 \times 10^{4} \mathrm{~g} \mathrm{~mol}^{-1}, M_{\mathrm{w}} / M_{\mathrm{n}}: 3.3\right)$ (C) and its fractionated components with different molecular weights (D) (eluent, chloroform; polystyrene standards). (E, F) Molecular weight dependency of the CD and absorption spectra of poly-9 in chloroform/acetonitrile $(60 / 40, \mathrm{v} / \mathrm{v})(\mathrm{E})$ and chloroform $(\mathrm{F})$ at $25^{\circ} \mathrm{C}$. To allow comparison of the $\mathrm{CD}$ intensities, the CD spectra were normalized with respect to the absorbance at the absorption maximum wavelength. The corresponding CD (unprocessed) and absorption (not normalized) spectra are shown in Fig. S6.† 
Cotton effect are most likely attributed to chiral exciton coupling between the adjacent bithiophene-based $\pi$-conjugated units, which are arranged in a clockwise twisting manner. This result indicates that the $\mathrm{CD}$ absorption of poly-9 in chloroform is mainly due to the central chirality of the glucose units; in other words, the poly-9 backbone did not form the specific higher-order structure in chloroform. This reasoning is also supported by the fact that the CD intensity of poly-9 in chloroform was almost independent of the temperature (ESI Fig. S4A $\mathrm{S}^{\mathrm{i}}$. In contrast, when the CD spectrum of poly-9 was measured in chloroform/acetonitrile mixed solvents, its CD intensity increased with an increase in the acetonitrile content. ${ }^{14}$ A clear hypochromic effect was also observed in the absorption spectra of poly-9. Considering the fact that a concentration dependence was not observed in the range of 0.01-1.0 mM (ESI Fig. S5 $\dagger$ ), the solvent-dependent CD spectral change observed for poly-9 could be ascribed to the conformational change within a single polymer chain, rather than to the formation of polymer aggregates through intermolecular interactions. In sharp contrast, the $\mathrm{CD}$ and absorption spectra of mono-10 did not show a clear solvent dependency (Fig. 2B). Based on the above results, we concluded that poly- 9 behaves as a foldamer and can change its conformation in response to the exterior environments. Poly-9 most likely folds into a preferred-handed helical structure in solutions containing high amounts of the poor solvent acetonitrile, whereas the polymer conformation becomes random in the good solvent chloroform. As is often the case with foldamer systems, ${ }^{2 b, h}$ a lyophobic interaction between aromatic units seems to be a critical factor for promoting helical folding and stabilizing the helical structure. The new CD bands around 340 and $460 \mathrm{~nm}$, which appear only in the helically folding state, are probably due to chiral exciton coupling derived from a helically twisting intramolecular $\pi$-stacked structure. Although the helical conformation of poly-9 formed in a mixed solvent system tended to collapse with increasing temperature, the CD intensity was maintained to some extent even at $55^{\circ} \mathrm{C}$ (ESI Fig. S4B $\dagger$ ).

\section{Influence on the molecular weight}

To investigate the influence of the molecular weight on the chiroptical properties, five samples of poly-9 with different molecular weights were prepared by SEC fractionation, and their CD and absorption spectra were measured in chloroform and a chloroform/acetonitrile mixture (60/40, v/v) (Fig. 2C-F). ${ }^{15}$ It was found that, in a chloroform/acetonitrile mixture (60/40, $\mathrm{v} / \mathrm{v}$ ), the magnitude of the CD absorption tended to increase with increasing molecular weight and reached an almost constant value when the molecular weight was more than $2.4 \times$ $10^{4} \mathrm{~g} \mathrm{~mol}^{-1}$ (Fig. 2E). This means that a cooperative intramolecular interaction along $c a .25$ repeating units, which is thought to correspond to about eight turns of the helix according to our computational study described below, is required to sufficiently stabilize the helically folded state of poly-9 in a chloroform/acetonitrile mixture $(60 / 40, \mathrm{v} / \mathrm{v})$. This also suggests that small molecular weight components should be excluded
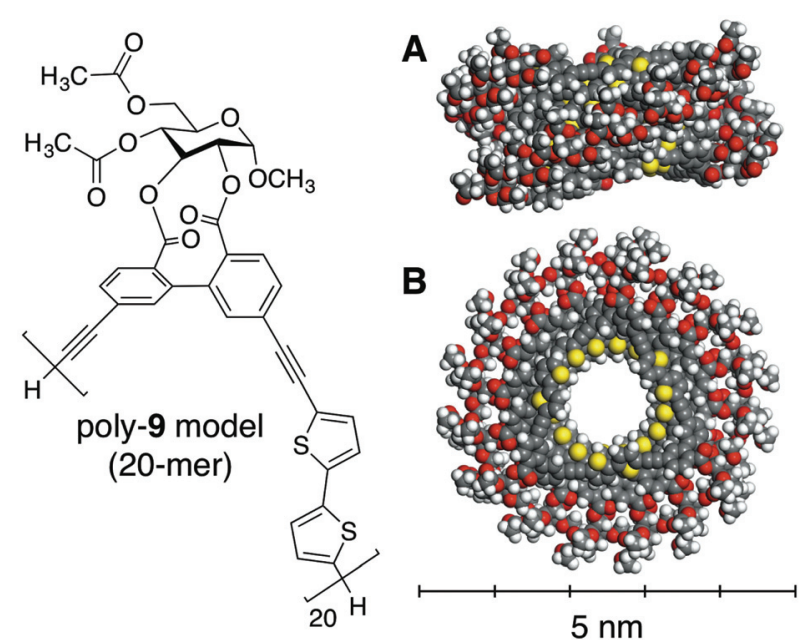

Fig. 3 Side view (A) and top view (B) of a possible left-handed helical structure of a poly- 9 model $(20$ mer). The structure is shown using the space-filling model.

to dissociate the information on helically folded poly-9 from that on the random-coil conformation. However, owing to the difficulty of obtaining a high molecular weight component in bulk, the as-synthesized poly- 9 was used in the subsequent study. As expected from the structural features of random polymer chains, the (chir)optical properties of poly-9 in chloroform were almost completely unaffected by the molecular weight (Fig. 2F).

Based on a molecular mechanics study for the model polymer with 20 repeating units (Fig. 3), ${ }^{16}$ the most stable conformation of poly-9 was estimated to be a left-handed helical shape with an interior cavity of $1.0 \mathrm{~nm}$, a helical pitch of $0.36 \mathrm{~nm}$ and approximately three repeating units per turn of the helix (ESI Fig. S7 and S8†). This is a possible structure for poly-9 in the solution containing plenty of acetonitrile. The calculated helical structure also revealed that the $\pi$-conjugated backbone of poly-9 is likely to be efficiently stacked between adjacent helical turns through intramolecular $\pi-\pi$ interactions (ESI Fig. S8†). This situation could explain the hypochromic effect observed in the helically folded state (see Fig. 2A).

\section{Importance of the biphenyl framework}

To demonstrate the importance of the glucose-linked biphenyl framework in the left-handed helix formation, the chiroptical properties of poly-11 without a biphenyl framework were compared with those of poly-9 (Fig. $2 \mathrm{~A}$ and $\mathrm{S} 9 \dagger$ ). It was found that the CD and absorption spectra of poly-11 in chloroform were very similar to those in the chloroform/acetonitrile $(60 / 40 \mathrm{v} / \mathrm{v})$ solution, and the intensities and patterns were almost the same as those observed for poly- $\mathbf{9}$ with a random conformation in chloroform. This suggests that poly-11 did not show a solvent-dependent conformational change; this is probably because the poly-11 backbone is too flexible to form a regular higher-order structure. This result suggests that the biphenyl framework linked to a glucose unit provides a moderate rigid- 
ity to the main chain, and plays a crucial role in the construction of the specific higher-order structure.

\section{Chiroptical properties in film-states}

We next investigated the film-state chiroptical properties of poly-9. A poly-9 film was prepared by spin coating its chloroform or chloroform/acetonitrile $(60 / 40, \mathrm{v} / \mathrm{v})$ solution onto a quartz plate, and the two CD spectra are depicted in Fig. 4A. The influence of the optical anisotropy was evaluated prior to measuring the film-state CD spectra, and was found to be negligible (ESI Fig. S11†). Compared with the chloroform-cast film, the film prepared from the mixed solvent system showed a more intense CD signal. This trend is consistent with the result observed for the corresponding solution states, although the $\mathrm{CD}$ intensity ratio and absorption regions in the film states are slightly different from those in the solution states. This indicates that the random and helical conformations formed in the solution states are basically maintained in the solid
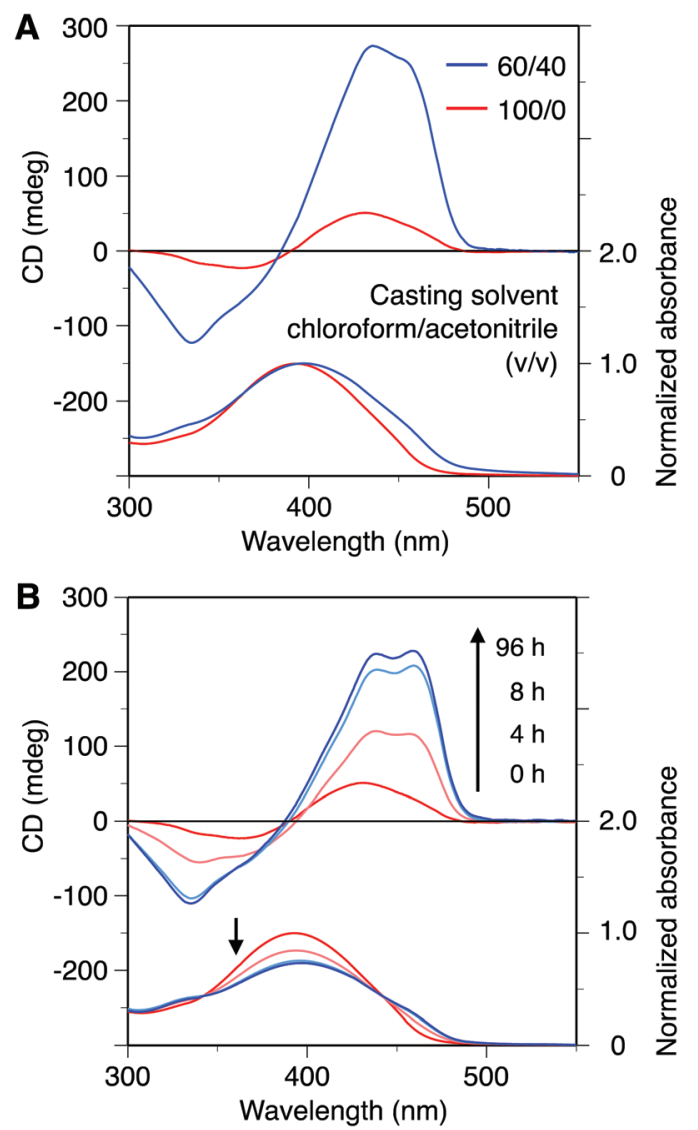

Fig. 4 (A) $C D$ and absorption spectra of poly-9 in the film states at room temperature. The spectra indicated by red and blue lines were obtained from the spin-cast poly- 9 films prepared from chloroform and chloroform/acetonitrile $(60 / 40, \mathrm{v} / \mathrm{v})$ solutions, respectively. To allow comparison of the $C D$ intensities, the $C D$ spectra were normalized with respect to the absorbance at the absorption maximum wavelength. (B) Time-dependent CD spectral changes of a chloroform-cast film of poly9 on exposure to acetonitrile vapor. The corresponding CD (unprocessed) and absorption (not normalized) spectra are shown in Fig. S10.† states. The CD intensities of the poly-9 cast films were larger than those observed for the corresponding solution states, particularly in the mixed solvent system (ESI Fig. S12 $\dagger$ ). This implies that poly-9 may be more likely to fold into a helical conformation in the solid state than in solution, probably because the $\pi-\pi$ interactions are more effective in the solid state as a result of the suppressed mobility of the polymer chain. Interestingly, when the chloroform-cast poly-9 film was exposed to acetonitrile vapor at room temperature, its (chir) optical properties gradually changed with exposure time, and a remarkable increase in the $\mathrm{CD}$ intensity and a hypochromic effect in the absorption were observed (Fig. 4B). After $96 \mathrm{~h}$ exposure, the CD intensity reached a higher value than that of the cast film prepared from the chloroform/acetonitrile (60/40, v/v) solution (ESI Fig. S13†). This result clearly suggests that a conformational transition of the poly-9 main chain from random-coil to helical structures can be achieved in the film state through solvent vapor annealing.

\section{Circularly polarized luminescence properties}

Poly-9 is optically active and can emit an apparent fluorescence under irradiation with near-ultraviolet light owing to its bithiophene-based $\pi$-conjugated backbone. The relationship between the CPL properties and the main-chain conformations of poly9 was therefore investigated with the aim of developing a foldamer-based advanced material capable of changing its performance through a folding/unfolding transition. The PL and CPL spectra of poly-9, mono-10 and poly-11 in solution are shown in Fig. 5A and S15. $\uparrow$ Photographs of the solutions under irradiation at $365 \mathrm{~nm}$ are depicted in Fig. S16. $\dagger$ Poly-9 showed a green or blue emission, derived from the $\pi$-conjugated backbone, in chloroform/acetonitrile $(60 / 40, \mathrm{v} / \mathrm{v})$ or chloroform, respectively (ESI Fig. S16A $\dagger$ ). In contrast, the PL colors of mono-10 and poly-11 in chloroform were similar to those in the mixed solvent (ESI Fig. S16B and $\mathrm{C}^{\dagger}$ ). The solvent-dependent PL color change observed for poly- $\mathbf{9}$ is considered to arise from the folding/unfolding behavior, which is accompanied by a change in the $\pi$-conjugation length of the main chain. The fluorescence quantum yield of poly-9 in chloroform/acetonitrile $(60 / 40, \mathrm{v} / \mathrm{v})$ was determined to be $6 \%$, which is smaller than that in chloroform (13\%) (ESI Fig. S16A $\dagger$ ). This fluorescence quenching is maybe due to a tight $\pi-\pi$ interaction in the intramolecular $\pi$-stacked structure (ESI Fig. S8 †). Poly-9 showed a clear CPL emission, the intensity of which was dependent on the solvent used. Compared with the result in chloroform, poly-9 exhibited superior CPL emission in a chloroform/acetonitrile $(60 / 40, \mathrm{v} / \mathrm{v})$ mixture, and the maximum value of its CPL dissymmetry factor $\left(g_{\text {lum }}=2\left(I_{\mathrm{L}}-I_{\mathrm{R}}\right) /\left(I_{\mathrm{L}}+I_{\mathrm{R}}\right)\right.$, where $I_{\mathrm{L}}$ and $I_{\mathrm{R}}$ are the PL intensities of the left- and righthanded circularly polarized light, respectively) was estimated to be $4.5 \times 10^{-3}$. This value is 15 times higher than that calculated for the chloroform solution $\left(g_{\text {lum }}=0.3 \times 10^{-3}\right)$. As expected from the results of the CD spectral analysis, the CPL properties of mono-10 and poly-11 were almost independent of the chloroform/acetonitrile mixing ratio, and their $g_{\text {lum }}$ values were determined to be $0.4 \times 10^{-3}$ and $0.6 \times 10^{-3}$, 

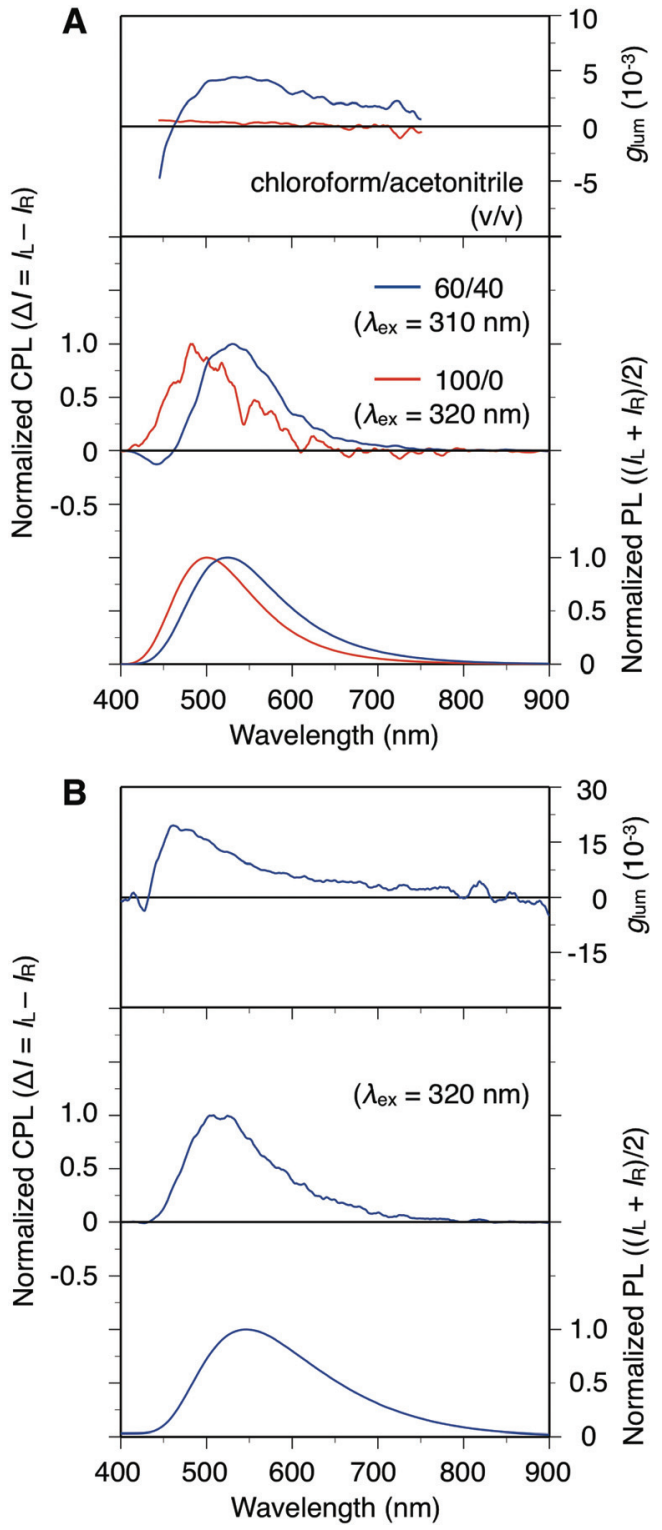

Fig. $5 \mathrm{PL}$ (bottom), CPL (middle) and $g_{\text {lum }}$ (top) spectra of poly-9 (1.0 $\times$ $\left.10^{-4} \mathrm{M}\right)$ in chloroform/acetonitrile $(100 / 0$ or $60 / 40, v / v)(A)$ and of a chloroform-cast film of poly-9 after exposure to acetonitrile vapor for $96 \mathrm{~h}(\mathrm{~B})$ at room temperature. The corresponding PL (not normalized) and CPL (unprocessed) spectra are shown in Fig. S14. $\dagger$

respectively, even in the chloroform/acetonitrile $(60 / 40, \mathrm{v} / \mathrm{v})$ mixture (ESI Fig. S15 $\dagger$ ). These values are comparable to the result for random-coiled poly-9 in chloroform. The above results suggest that the helically folded state is an important requirement for efficient CPL emission. In addition, we found that after exposure to acetonitrile vapor, the poly- 9 film had a $g_{\text {lum }}$ value of $1.9 \times 10^{-2}$, as shown in Fig. 5B and S17. $\uparrow$ This $g_{\text {lum }}$ value is sufficiently high compared with those of previously reported one-handed helical polymers in molecularly dispersed solutions and non-oriented films. ${ }^{17}$ The intermolecular couplet of the folding poly-9 may also contribute to the achievement of a high $g_{\text {lum }}$ value.

\section{Conclusions}

We have synthesized an optically active diethynyl compound (GLB-7) containing a structural element of the naturallyoccurring ellagitannins, which has a preferred-handed axially twisted biphenyl group attached to a saccharide unit through ester linkages. Polymerization of GLB-7 with a bithiophenebased cross-coupling partner provided a $\pi$-conjugated polymer (poly-9), which shows a solvent-induced conformational change. Based on a comparison of the chiroptical properties of poly-9 and model compounds (mono-10 and poly-11), we can conclude that poly-9 behaves as a foldamer, and that its main chain folds into a preferred-handed helical conformation in solution and the solid state under an appropriate exterior environment. This consideration is further supported by a molecular modeling study. We also found that poly-9 can efficiently emit left-handed circularly polarized light mainly due to its helical chirality, and a $g_{\text {lum }}$ value of $1.9 \times 10^{-2}$ was achieved in the film state. We believe that this research demonstrates a new possibility for the development of future functional materials using the chirality of abundant biomass resources.

\section{Acknowledgements}

This work was supported by the Japan Society for the Promotion of Science (JSPS) KAKENHI Grants-in-Aid for Scientific Research (C), Grant No. 26410129 and the Ogasawara Foundation for the Promotion of Science \& Engineering.

\section{Notes and references}

1 (a) H. Satomi, K. Umemura, A. Ueno, T. Hatano, T. Okuda and T. Noro, Biol. Pharm. Bull., 1993, 16, 787-790; (b) L. Boulekbache-Makhlouf, E. Meudec, M. Chibane, J.-P. Mazauric, S. Slimani, M. Henry, V. Cheynier and K. Madani, J. Agric. Food Chem., 2010, 58, 12615-12624.

2 (a) S. H. Gellman, Acc. Chem. Res., 1998, 31, 173-180; (b) D. J. Hill, M. J. Mio, R. B. Prince, T. S. Hughes and J. S. Moore, Chem. Rev., 2001, 101, 3893-4011; (c) Foldamers: Structure, Properties, and Applications, ed. S. Hecht and I. Huc, Wiley-VCH, Weinheim, 2007; (d) B. Gong, Acc. Chem. Res., 2008, 41, 1376-1386; (e) I. Saraogi and A. D. Hamilton, Chem. Soc. Rev., 2009, 38, 1726-1743; $(f)$ G. Guichard and I. Huc, Chem. Commun., 2011, 47, 5933-5941; (g) D. W. Zhang, X. Zhao, J. L. Hou and Z. T. Li, Chem. Rev., 2012, 112, 5271-5316; (h) C. S. Hartley, Acc. Chem. Res., 2016, 49, 646-654.

3 (a) J. C. Nelson, J. G. Saven, J. S. Moore and P. G. Wolynes, Science, 1997, 277, 1793-1796; (b) R. B. Prince, J. G. Saven, P. G. Wolynes and J. S. Moore, J. Am. Chem. Soc., 1999, 121, 3114-3121.

4 (a) M. S. Gin, T. Yokozawa, R. B. Prince and J. S. Moore, J. Am. Chem. Soc., 1999, 121, 2643-2644; (b) E. Kolomiets, V. Berl and J. M. Lehn, Chem. - Eur. J., 2007, 13, 5466-5479; 
(c) H.-Y. Hu, J.-F. Xiang, Y. Yang and C.-F. Chen, Org. Lett., 2008, 10, 69-72; (d) T. Kaneko, H. Abe, M. Teraguchi and T. Aoki, Macromolecules, 2013, 46, 2583-2589; (e) H. Okuda, Y. Koyama, S. Uchida, T. Michinobu, H. Sogawa and T. Takata, ACS Macro Lett., 2015, 4, 462-466.

5 (a) L. Brunsveld, R. B. Prince, E. W. Meijer and J. S. Moore, Org. Lett., 2000, 2, 1525-1528; (b) R. B. Prince, L. Brunsveld, E. W. Meijer and J. S. Moore, Angew. Chem., Int. Ed., 2000, 39, 228-230; (c) A. Tanatani, A. Yokoyama, I. Azumaya, Y. Takakura, C. Mitsui, M. Shiro, M. Uchiyama, A. Muranaka, N. Kobayashi and T. Yokozawa, J. Am. Chem. Soc., 2005, 127, 8553-8561; (d) R. W. Sinkeldam, F. J. M. Hoeben, M. J. Pouderoijen, I. De Cat, J. Zhang, S. Furukawa, S. De Feyter, J. A. J. M. Vekemans and E. W. Meijer, J. Am. Chem. Soc., 2006, 128, 16113-16121; (e) R. M. Meudtner and S. Hecht, Angew. Chem., Int. Ed., 2008, 47, 4926-4930; $(f)$ Y. Wang, F. Li, Y. Han, F. Wang and H. Jiang, Chem. - Eur. J., 2009, 15, 9424-9433; (g) M. Banno, T. Yamaguchi, K. Nagai, C. Kaiser, S. Hecht and E. Yashima, J. Am. Chem. Soc., 2012, 134, 8718-8728; (h) R. Pfukwa, P. H. J. Kouwer, A. E. Rowan and B. Klumperman, Angew. Chem., Int. Ed., 2013, 52, 1104011044; ( $i$ ) H. Sogawa, Y. Miyagi, M. Shiotsuki and F. Sanda, Macromolecules, 2013, 46, 8896-8904.

6 (a) C. R. Woods, M. Benaglia, J. S. Siegel and F. Cozzi, Angew. Chem., Int. Ed. Engl., 1996, 35, 1830-1833; (b) Y. Inai, Y. Ishida, K. Tagawa, A. Takasu and T. Hirabayashi, J. Am. Chem. Soc., 2002, 124, 2466-2473; (c) J. Clayden, A. Lund, L. Vallverdú and M. Helliwell, Nature, 2004, 431, 966-971; (d) H. Jiang, C. Dolain, J.-M. Léger, H. Gornitzka and I. Huc, J. Am. Chem. Soc., 2004, 126, 1034-1035; (e) Z. Dong, R. J. Karpowicz, S. Bai, G. P. A. Yap and J. M. Fox, J. Am. Chem. Soc., 2006, 128, 14242-14243; (f) H. Abe, D. Murayama, F. Kayamori and M. Inouye, Macromolecules, 2008, 41, 6903-6909; (g) A. M. Kendhale, L. Poniman, Z. Dong, K. Laxmi-Reddy, B. Kauffmann, Y. Ferrand and I. Huc, J. Org. Chem., 2011, 76, 195-200.

7 (a) E. Yashima, K. Maeda, H. Iida, Y. Furusho and K. Nagai, Chem. Rev., 2009, 109, 6102-6211; (b) A. Roy, P. Prabhakaran, P. K. Baruah and G. J. Sanjayan, Chem. Commun., 2011, 47, 11593-11611; (c) M. Yamaguchi, M. Shigeno, N. Saito and K. Yamamoto, Chem. Rec., 2014, 14, 15-27.

8 (a) V. Maurizot, C. Dolain and I. Huc, Eur. J. Org. Chem., 2005, 1293-1301; (b) C. Li, G. T. Wang, H. P. Yi, X. K. Jiang, Z. T. Li and R. X. Wang, Org. Lett., 2007, 9, 1797-1800; (c) K. Maeda, K. Morioka and E. Yashima, Macromolecules, 2007, 40, 1349-1352; (d) H. Juwarker, J.-m. Suk and
K.-S. Jeong, Chem. Soc. Rev., 2009, 38, 3316-3325; (e) H. Abe, K. Okada, H. Makida and M. Inouye, Org. Biomol. Chem., 2012, 10, 6930-6936; (f) Y. Ferrand, N. Chandramouli, A. M. Kendhale, C. Aube, B. Kauffmann, A. Grélard, M. Laguerre, D. Dubreuil and I. Huc, J. Am. Chem. Soc., 2012, 134, 11282-11288; $(g)$ Z. Chen, Q. Wang, X. Wu, Z. Li and Y. B. Jiang, Chem. Soc. Rev., 2015, 44, 4249-4263.

9 N. Ousaka, T. Yamaguchi and E. Yashima, Chem. Lett., 2014, 43, 512-514.

10 H. Noguchi, M. Takafuji, V. Maurizot, I. Huc and H. Ihara, J. Chromatogr., A, 2016, 1437, 88-94.

11 T. Itoh, J.-i. Chika, S. Shirakami, H. Ito, T. Yoshida, Y. Kubo and J.-i. Uenishi, J. Org. Chem., 1996, 61, 3700-3705.

12 (a) G. Capozzi, C. Ciampi, G. Delogu, S. Menichetti and C. Nativi, J. Org. Chem., 2001, 66, 8787-8792; (b) D. S. Surry, X. Su, D. J. Fox, V. Franckevicius, S. J. F. Macdonald and D. R. Spring, Angew. Chem., Int. Ed., 2005, 44, 1870-1873.

13 The normally distributed peak shapes in the chromatograms indicate that the isomerization hardly proceeded during the separation. However, reinjection of GLB-7 $\boldsymbol{R}$ immediately after fractionation, resulted in a chromatogram containing GLB-7S and GLB-7 $\boldsymbol{R}$ as major and minor components, respectively. These results suggest that the isomerization occurs outside the column and may be accelerated by exposure to light. A detailed study of the diastereomeric isomerization of GLB-7S and GLB-7 $\boldsymbol{R}$ is currently in progress and will be published elsewhere.

14 We could not perform spectral measurements using mixed solvents containing more than 50 vol\% acetonitrile because of the insolubility of poly-9.

15 Because of the difficulty in controlling the concentration of each fractionated sample, CD spectra normalized with respect to the absorbance at the absorption maximum wavelength are shown.

16 The model compound containing acetyl groups instead of [2-(2-methoxyethoxy)ethoxy]acetyl groups was used for the computational study as a simplified model.

17 (a) K. Suda and K. Akagi, Macromolecules, 2011, 44, 94739488; (b) K. Watanabe, T. Sakamoto, M. Taguchi, M. Fujiki and T. Nakano, Chem. Commun., 2011, 47, 10996-10998; (c) D. Lee, Y.-J. Jin, H. Kim, N. Suzuki, M. Fujiki, T. Sakaguchi, S. K. Kim, W.-E. Lee and G. Kwak, Macromolecules, 2012, 45, 5379-5386; (d) Y. Nagata, T. Nishikawa and M. Suginome, Chem. Commun., 2014, 50, 9951-9953; (e) T. Shiraki, Y. Tsuchiya, T. Noguchi, S. Tamaru, N. Suzuki, M. Taguchi, M. Fujiki and S. Shinkai, Chem. - Asian J., 2014, 9, 218-222. 